\title{
Keyword index to volume 9
}

11 p15 region 409

$16 q$ partial trisomy 881

$17 \mathrm{p} 11.2892$

2qter deletion 881

$\alpha$-N-Acetylgalacto-saminidase 91

$\alpha$-NAGA deficiency 91

$\alpha_{1}$-antitrypsin enhancer polymorphism 273

A3243G 917

A7445G 56

ACE 797

ADPKD 957

ADULT syndrome 642

Affected sib-pairs 938

Age at onset 867

Ageing 877

Alagille syndrome 209

Allergy 945

Alpha globin 217

Alu 143

Alu insertion 267

Alzheimers 437

Alzheimers disease 279, 464, 802

Amyloglucosidase 388

Analphoid marker chromosome 572

Ancient DNA 185

Aneuploidy 395, 533

Angelman syndrome 519

Angiotensin converting enzyme 797

Arthritis 849

Association 437

Association analysis 130, 860

Asthma 341, 347, 867

$A T B^{O} / \mathrm{SLC} 1 \mathrm{~A} 5$ gene 860

Atherosclerosis 375

Atherosclerosis genes 364

Athletes 797

Atopy 347

ATR-16 217

Austria 226

Autism 556

Autoimmune 938

Automated genotyping 319

Autosomal dominant 39

Autosomal dominant inheritance 452

AXIN1 217
BAC contig 364, 501

Band 16p13.3 217

Beckwith-Wiedemann syndrome 409

Behçets disease 191

BIAcore 204

Biotinidase deficiency 237

Bipolar affective disorder 39

Brain 279

Brain structure 153

BRCA1 gene 787

BRCA1 424

BRCA2 424, 773

BRCA1/BRCA2 492

Breast cancer 953

Breast neoplasm 787

\section{C\&IT 953}

C282Y 843

Cadherin 197

CAG repeat disorder 431

CAG repeat expansion 160

CAL 953

Cancer 492, 811

Candidate gene 469

Candidate region 945

Cardiovascular disease 583

Carrier rate 634

Caste 794

Caucasian 577

Caucasus populations 267

cDNA cloning 922

Celiac disease 938

Celtic people 445

Censored traits 301

Charcot-Marie-Tooth neuropathy 646

Cholesterol 45, 375

Chromosomal band 11q23 121

Chromosome 1747

Chromosome 14802

Chromosome 17892

Chromosome 18922

Chromosome 191

Chromosome 1p21-p22 34

Chromosome 20209

Chromosome $3 q 452$

Chromosome 3q22 165

Chromosome 9501
Chromosome aberration rate 539

Chromosome mapping 328

Chromosome rearrangement(s) 319, 395

Chromsome 16217

CLCN1 903

Cleft lip and palate 747

Cleft palate 747

Clone end-sequencing 307

Comparative genomic hybridrisation (CGH) 539

Complete gene structure 561

Complex disease(s) 291, 316, 938

Complex III 510

Concentration 780

Confocal microscopy 815

Connexin 26226

Contig 892

Contig map 731

Continuous gene syndrome 892

Covariates 613

Creutzfeldt-Jakob disease 965

Crohns disease 627, 731

CTG repeats 638

Culture failures 539

Currarino syndrome 599

Cystathionine $\beta$-synthase (CBS) 583

Cystic fibrosis 273, 860

Cytochrome b 510

D8S2611 469

DASH 437

DCX 1

Deafness 917

Deletion 143

Dementia 802

Denaturing gradient gel electrophoresis 237

Depletion 279

Desmoglein 197

Desmosome 197

DFNA18 165

DGGE 957

DGGE analysis 556

Diabetes 917

Diet 375

Dilated cardiomyopathy 204, 311 
Dispersed repeats 143

DM 829

DM1 638

DM2 locus 165

DNA polymorphism 765

DNA-binding specificity 599

DNA-methylation 519

Dominance 197

Downs syndrome 404, 811

DRPLA 829

DYS388 22

DYS392 22

Dystonia 160

Ectodysplasin 355

ED1 gene 355

EEC syndrome 642

ELISA 811

Embryonal tumour 409

Emery-Dreifuss muscular dystrophy 204

Endostatin 811

Epidemiology 1

Epidermis 197

ER $\beta 802$

EST expression analysis 307

Evaluation 953

Evolution 765

Exon mapping 922

Exon skipping 113

Experimental arthritis 458

Experimental auto-immune encephalomyelitis 458

Factor VII deficiency 105

Familial defective apolipo-protein B-100 (FDB) 836

Familial hypercholes-terolaemia $(\mathrm{FH})$ 244, 815

Familial Mediterranean fever 45, 191

Faroe islands 388

FBAT 301

Fibrillin 13

Fine mapping 731

Finland 424

First trimester trisomy screening 404

FISH 395, 527, 533

FMF 473, 553, 634, 849

Founder effect 431, 638, 787

Founder mutation 424, 773

Founder populations 97

Fragile $X$ syndrome 153

French population 464

Gastric cancer 548

Gastritis 548
Gastrointestinal system 860

GC content 590

Gene 802, 965

Gene frequency 855

Gene structure 253

General pedigrees 335

Genetic 965

Genetic diversity 97

Genetic drift 634

Genetic heterogeneity 135, 867, 903

Genetic identification 185

Genetic linkage 458, 606, 646

Genetic predisposition to disease 328

Genetic service 244

Genetic susceptibility 458

Genetic testing 492

Genetics 375, 758, 945, 857

Genome scan 753, 945

Genome screens 621

Genome-wide scan 758, 938

Genome-wide search 646

Genomic imprinting 82

Genotype 849

Genotype error 130

Genotype risks 867

Genotype-phenotype correlation 13, 599

GJB2 226

Glaucoma 452

GLC1C 452

Glomangioma 34

Glutamate dehydrogenase 931

Glycine receptor 873

Glycogen debranching enzyme 388

Glycogen storage disease 388

Gm allotypes 765

GRB10 82

Guidelines 484

H63D 843

H63D mutation 961

Haemochromatosis 445, 961

Haemorrhagic disorder 105

Hamartin 823

Haploinsufficiency 217, 892

Haplotype 385, 638, 787

Haplotype analysis 464

Haplotype sharing 34

Haplotypes 291

Hearing impairment 56

Hearing loss 147, 226, 385

Hemochromatosis 843

Hereditary 135

Hereditary ataxia 160

Hereditary breast cancer 773
Hereditary hearing impairment 165

Hereditary paraganglioma 121

Heteroduplex single strand confirmational polymorphism (HEX-SSCP) 419

Heteroplasmic 917

HFE 843

HFE gene 961

HFE mutations 445

Hirschsprungs disease 419

HIV resistance 794

HLA-DR 341

HLA 590

HLXB9 mutations 599

Homocysteine 583

Homozygosity mapping 375, 561, 753

Human 510

Human chromosome 2039

Human genetic diversity 765

Hyper IgD syndrome 260

Hyperammonaemia 931

Hyperekplexia 873

Hyperhomocysteinaemia 583

Hyperimmunoglobulinaemia $D$ and periodic fever syndrome 253

Hyperinsulinism 931

Hypodontia 743

Hypoglycaemia 931

Iberian peninsula 843

IBM2 501

$\operatorname{lgD} 849$

IgG immunoglobulins 765

IGHG3 765

Imprinting 121, 519

Inborn errors 253

Inflammatory bowel disease 328, 627

Insertion 143

Interphase FISH 286

Intestinal metaplasia 548

Intrauterine survival 286

In vitro mutagenesis 204

In vivo assay 815

Ion channels 873

JAG1 mutation 209

KCNQ10T gene 409

Kennedy disease 431

Keratoderma 197

Knockout mouse 153

Lebanese 51

Leber congenital amaurosis 561

Limb abnormality 642 
Linkage 328, 375, 621, 627, 758, 867

Linkage analyses 39

Linkage analysis $130,135,165,452$, $613,747,753,855,938$

Linkage disequilibrium 291, 301, 464, 590,731

Lip pits 747

Locus 945

Lod score 328

Logistic residuals 613

LOH 121

Louis XVII 185

Low-density lipoprotein receptor 244

LRP 464

Magnetic resonance imaging 153

Males 492

Manic-depressive illness 39

MAPH 527

Mapping 364

Marfan syndrome 13

Maternal heterodisomy 9914

Maternal inheritance 56

Maternal serum screening 404

Maximum-Likelihood-Binomial 613

MECP2 178

MECP2 gene 231, 556

MEFV 634

MEFV 191

$M E F V$ gene 45, 553

Meiotic segregation 395

MELAS 805

MELAS syndrome 59

Mental retardation 71, 153, 319, 527

Methylation 409

Methylmalonic acidemia 577

Methylmalonyl-CoA mutase 577

Mevalonate kinase 253, 260

Mevalonic aciduria 253

MG2 347

MHC 590

Microdeletion syndrome 892

Microsatellite repeats 328

Microsatellites 22

Mild phenotype 209

Missing genotypes 301

Mitochondral encephalomyopathies 59

Mitochondria 279

Mitochondrial 917

Mitochondrial diseases 510

Mitochondrial DNA 185, 311, 510

Mitochondrial DNA mutation 56

Mixed model 613

Molecular analysis 484

Monosomy 217
Morbidity 877

Mortality 877

Mosaicism 209

Mosaicism 887

MRX 71

MRXS 71

MtDNA 97, 147, 279, 805

MtDNA mutation 385

MUC1 548

MUC7 347

Mucin 347, 548

Multicolour banding (MCB) 572

Multiple epiphyseal dysplasia 606

Multiple glomus tumor 34

Multiple sclerosis 458, 621

Mutation 1, 147, 577, 849, 917

Mutation age estimation 773

Mutation analysis 873

Mutational predilection sites 599

Mutational spectra 45

Mutational spectrum 355

Mutations 113, 178, 191, 237, 253, 473, 553

Myeloperoxidase 780

Myoblast 204

Myotonia 903

Myotonia congenita 903

ND6 805

NEDD4 922

NEDD4L 922

Net reproduction rate 59

Neuroaxonal dystrophy 91

Neuromuscular diseases 501

Neuronal acetylcholine receptor 469

Neuropeptides 469

New mutation 836

NLS motif 599

Nonsense mutation 743

Nuclear lamina 204

OPA1 307

Oestrogen receptor $\beta 802$

Oligodontia 743

On-line database 71

Osteochondrodysplasia 606

Ovarian carcinoma 424

Ovarian neoplasm 787

Overgrowth syndrome 409

PAX9 743

PAX9 743

PAC contig 307

Pallister-Killian syndrome (PKS) 572

Parameter misspecification 855
Parental origin 231

Parternal isodisomy 21914

Partial tetrasomy $12 \mathrm{p}$ 572

Paternal age 533

PCap (Predisposing for Cancer Prostate) 135

Penetrance 63

Peopling of Europe 27

Periodic fever 260

Phenotype 849

Phenotype/genotype correlation 355

Phylogeny 765

Physical and transcript map 501

Physical map 121

PKD1 957

PKS critical region 572

Plasma TNFRSF1A levels 63

POAG 452

Polycystic kidney disease 957

Polyglutamine disease 160

Polymorphic markers 758

Polymorphism 347, 437, 469, 548, 780, 965

Polymorphisms 267, 501

Population 634, 965

Population frequencies 903

Population genetics 45, 59, 316, 829, 843

Population screening 27, 226

Pore-lining domain 873

Positional cloning 364, 731

Power 291, 335

Prader-Willi syndrome 519

Preeclampsia 758

Prenatal diagnosis 1, 404, 881

Prevalence 903

Prevalence in Poland 836

Prion 965

PRNP 965

Prostate cancer 135

Psychological distress 492

Psychomotor retardation 881

Pulmonary outcome 273

QTL 130

QTL linkage 335

Quantitative traits 341

Recombination 143, 590

Recurrent mutation 355

Replication 627

Reproduction 59

Retroposition 143 
RET proto-oncogene mutations 419

Rett syndrome 171, 178, 231, 556

Review 473

RC-TDT 301

Rhinitis 945

Ring X 171

Roma (gypsies) 97

RPGR-interacting protein (RPGR1P1) gene 561

Sardinians 621

SBMA 431

SCA1 loci 829

Schindler disease 91

Schizophrenia 469

Seckel syndrome 753

Selection 59

Serum 780

Sex ratio 533

SHFM 642

Short tandem repeat 590

Short tandem repeat polymorphism 829

Sib-pair 627, 758

Silver-Russel syndrome 887

Single base-pair mutation 105

Single nucleotide polymorphism 191

Skeletal dysplasia 606

Skewed $\mathrm{X}$ inactivation 887

SLOS 45

SMA 484

Smith-Magenis syndrome 892

SMN 484

SNP 130, 437

SNP genotyping 316

Sperm chromosome 395
Sperm nuclei 395

Spermatozoa 533

Spinal muscular atrophy 113

Spontaneous abortions 539, 914

Sporadic 437

Sport 797

SRS 82

Stepwise mutation model 22

Stop codon mutations 823

Subcortical band heterotopia 1

Subtelomeric 527

Subtelomeric translocation 881

Survival motor neuron gene 113

Susceptibility 548

Synteny 458

Tail vein injection 815

Taiwan 638

TATA-binding protein 160

TDT 301

Teaching 953

Telomere 319

Telomere restriction fragment length 877

Temperature-gradient gel electrophoresis (TGGE) 13

Time lapse 815

Tirol 226

Tissue-specific 82

TNFRSF1A mutation 63

Tooth agenesis 743

TP63 642

Transcription map 892

Transfer RNA ${ }^{\text {Ser(UCN) }} 385$

TRAPS 63
Tribe 794

Trinucleotide repeat 431

Triple test 1

Trisomic rescue 286

Trisomy 13286

Trisomy 18286

Trisomy 21 incidence 1

Trisomy 7887

TRNA ${ }^{\text {Leu(CUN) }} 311$

TRNASer $^{(\mathrm{UCN})} 56$

Tuberin 823

Tuberous sclerosis 823

Turkey 965

Ulcerative colitis 627

Ullrich-Turner syndrome 877

Unaffected 613

Uniparental disomy 319, 914

Van der Woude syndrome 747

Variance components 335

Variation 316

Variation factors 780

Vascular anomaly 34

VNTR 583

X chromosome 71

$X$ inactivation $1,171,178$

X-linked hydrocephalus 153

XLMR 71

XLIS 1

Y chromosome 22, 27, 97 